\title{
LE CORPS PHOTOGRAPHIQUE DE L'UNIVERSITÉ ${ }^{1}$
}

\author{
Jan Baetens et Hilde Van Gelder ${ }^{2}$
}

\section{De l'image à l'album}

Toute organisation, qu'elle se constitue ou qu'elle se transforme, tend à créer, tant pour elle-même que pour le monde extérieur, une représentation de $\operatorname{soi}^{3}$. De la même façon qu'elle pense, et partant récrit, sans cesse sa propre histoire, elle développe aussi des stratégies pour gérer sa propre image, c'est-à-dire la représentation visuelle de l'idée qu'elle se fait et cherche à donner d'elle-même. S'il est aussi ancien que les institutions elles-mêmes ${ }^{4}$, le parti pris de l'image est évidemment renforcé par la révolution visuelle de notre culture et la transformation de nos sociétés en sociétés de spectacle. Il n'en va pas autrement pour les universités qui, à l'instar de n'importe quelle autre

1 Les auteurs tiennent à remercier vivement M. Mark Derez de l'aide apportée lors de la préparation, puis de la rédaction de ce texte.

2 Lieven Gevaert Centre for Photography and Visual Studies (KU Leuven) (www.lievengevaertcentre.be).

3 Voir Recherches en communication, $\mathrm{n}^{\circ} 17$ ("Esthétique des organisations"), 2002.

4 Pour quelques exemples de l'époque classique, voir entre autres Jean-Marie APOSTOLIDĖS, Le roi-machine: spectacle et politique au temps de Louis XIV, Paris, Éd. de Minuit, 1981, et Louis Marin, Le portrait du roi, Paris, Éd. de Minuit, 1987. 
institution, interrogent à la fois leur propre histoire et leur propre image, passée mais aussi présente.

Pareille autoreprésentation, sans laquelle l'institution n'existerait pas, peut prendre évidemment les formes les plus diverses. Celles-ci, pourtant, ne font pas que reproduire passivement quelque idée préalable. D'une part, toute représentation implique en effet une part de construction, consciente ou non, volontaire ou non : si plate qu'elle soit, une image n'est jamais neutre. D'autre part, toute représentation dépend aussi du média qui l'actualise et qui impose souvent sa propre logique interne à celle des idées qu'on veut lui faire signifier : si docile qu'il puisse paraître, un média n'est jamais totalement transparent. L'un et l'autre de ces phénomènes, le conflit entre reproduction et construction d'un côté, la tension entre l'idée et la matérialité de l'autre, expliquent la grande dynamique des représentations institutionnelles, écartelées qu'elles sont entre modes et exigences contradictoires. L'analyse de quelques albums photographiques réalisés par l'université de Louvain depuis la fin du XIX ${ }^{\mathrm{e}}$ siècle jusqu'à nos jours fournira ici l'occasion d'étudier plus en détail certains des mécanismes et quelques-unes des mutations qu'entraîne la conversion visuelle concrète, plus particulièrement photographique, d'une idée ou d'une image abstraite'.

Il convient cependant de le spécifier tout de suite : l'album photographique, que l'on considère dans cet article comme un média spécifique, $n$ 'est pas une expansion mécanique de la photographie elle-même. Contrairement à celle-ci, l'album interdit en effet de concevoir ou d'élaborer des représentations uniques. L'image produite par un album ne sera donc jamais celle d'un logo par exemple, pour utiliser une terminologie contemporaine, c'est-à-dire d'une image unique qui condense à elle seule l'image ou les images abstraites que l'on cherche à communiquer; elle sera forcément de l'ordre de la série, ce qui introduit tout de suite des enjeux très particuliers. Qui plus est, la série en question n'est pas détachable d'un support déterminé, en l'occurrence le livre. L'observation peut

1 La focalisation exclusive de cet article sur les albums photographique explique l'absence, regrettable mais cohérente, de toute une série d'autres documents iconographiques, dont l'analyse devrait un jour compléter la présente lecture : par exemple un album de lithographies représentant un cortège historique en 1884 , l'étude (peu illustrée, mais historiquement capitale) de Victor BranTs, Coup d'reil sur l'université, de 1909, ou encore un album "virtuel " (jamais publié) consacré aux étudiants et professeurs morts pour la patrie en 1914-1918. 
paraître élémentaire, mais ses conséquences ne le sont nullement: avec le livre, la série se transforme en effet en séquence, c'est-à-dire en série vectorisée, voire temporellement orientée, conduisant d'un début à une fin; corollairement, le livre, qui est aussi une machine à générer du texte en marge et autour des images, superpose à la séquence visuelle toute une série de données verbales dont l'impact n'est jamais négligeable.

\section{De tout-Louvain au tout de Louvain}

En 1884, l'université de Louvain compose un album très grand format, environ $50 \mathrm{~cm}$ sur $75 \mathrm{~cm}$, répertoriant l'ensemble de son corps enseignant'. Faculté par faculté, à raison d'une feuille par unité, le lecteur reçoit un aperçu complet des professeurs dont les images en ovale, toutes de format et de style identiques, se disposent de façon circulaire sur la page autour d'une image centrale, celle, légèrement plus grande, du doyen ${ }^{2}$. En 2001, à l'occasion des festivités entourant les 575 ans de l'université, paraît du côté flamand ${ }^{3}$ un nouvel album, cette fois-ci de format plus réduit, proche du format commercial standard A4, avec une série de portraits réalisés par le photographe Jacques Sonck, bien connu pour ses autres séries de portraits souvent consacrés, un peu dans la lignée de Diane Arbus, à des personn(ag)es "bizarres"4. A la différence de l'album du XIX ${ }^{\mathrm{e}}$ siècle, les personnes photographiées appartiennent ici à toutes les catégories sociales, des étudiants aux femmes de ménage en passant par les techniciens, les infirmières, les habitants de la ville de Louvain même et ainsi de suite (le lien "direct" avec l'université au sens traditionnel est par moments assez ténu, ce qui devient pour cette raison même doublement significatif). Sans être absents du livre, bien entendu, les professeurs et

1 Université catholique de Louvain. Le corps professoral en 1884. Sowvenir des fêtes jubilaires 1834-1884, Louvain, 1884, publié par l'université.

2 Cette description fait l'impasse sur quelques détails, qui n'engagent pourtant pas le fond de l'analyse.

3 Pour la période post-1971, le présent article s'attache uniquement à l'iconographie de la KUL, pour des raisons qui tiennent au sujet même de ce texte : le rapport, par images interposées, entre l'université et son cadre urbain. Une analyse comparée avec l'iconographie de l'UCL serait passionnante et nécessaire, mais déborde le cadre de la présente analyse.

4 Jacques Sonck. Het gezicht van de KU Lewven, Leuven, 2001, publié par l'université. 
dignitaires de l'université ne se distinguent du reste pas toujours de manière très visible des autres classes de "sujets photographiques".

De prime abord, la comparaison de ces deux galeries de portraits, l'une et l'autre d'une grande homogénéité stylistique, dispose une leçon très simple, trop simple presque pour qu'on ose encore l'énoncer : en l'espace d'un siècle, l'université est descendue de sa tour d'ivoire, la démocratisation se reflétant non seulement dans le choix élargi des groupes sociaux qui posent devant la caméra, mais aussi et surtout dans leur traitement rigoureusement identique ${ }^{1}$ (cette analogie stylistique est néanmoins une arme très perverse, puisque l'on distingue rapidement que la photogénie et le glamour, pour ne rien dire de la sympathie du photographie, sont nettement plus présents du côté des "petits" que des "grands" de ce monde ${ }^{2}$ ).

Si elle n'est pas fausse, pareille conclusion reste pourtant bien trompeuse. Elle dissimule en effet une mutation bien plus importante, mais sans doute moins visible au regard des images isolées. En effet, ce qui change le plus radicalement de 1884 à 2001, c'est moins la nature ou le style des sujets photographiés que la structure d'ensemble de l'album. En 1884, cette structure est encore clairement unitaire et hiérarchique. Selon un modèle qu'on pourrait dire arborescent, on passe de l'ensemble (l'université) aux parties (les facultés), que l'on parcourt ensuite dans un ordre très mesuré qui est tout sauf indifférent (on commence évidemment par la faculté de théologie, pour terminer par les ingénieurs et les agronomes ; il faut noter du reste que l'importance de chaque faculté est inversement proportionnelle au nombre de professeurs qu'elle emploie: importance symbolique de la rareté, disqualification peu démocratique du plus grand nombre). A l'intérieur de chaque faculté, la même logique structurelle est à l'œuvre, puisque la photo du doyen, primus inter pares, apparaît au centre du cercle de ses collègues, que l'on imagine quant à eux réunis, sans autre distinction de rang ou d'ancienneté, autour de la "table ronde"

\footnotetext{
I A quelques exceptions près, puisque l'identité stylistique des images (tout le monde pose de la même façon devant le même décor, tout le monde est éclairé et photographié selon des angles et à des distances comparables) n'exclut pas des variations à hauteur des reproductions (certaines images occupent seules une page entière, d'autres sont imprimées en petit format et se donnent à voir par groupes de quatre). Quant à l'ordre d'apparition, non égalitaire par définition, force est de reconnaître qu'il ne privilégie guère ceux qui, plus de cent ans plus tôt, monopolisaient encore la totalité du champ visuel.

2 Selon, bien sûr, ce qu'il faut bien nommer une convention moderne devenue un peu lourde...
} 
de la faculté. Une place pour chaque chose, et chaque chose à sa place, voilà, en des termes peut-être un peu familiers, à quoi se ramène au fond cette logique arborescente, où l'individualité des portraiturés ne l'emporte certainement pas sur les fonctions revêtues. Tous les éléments de la structure obéissent aux mêmes principes, qui innervent l'ensemble de haut en bas et de bas en haut.

L'album de Jacques Sonck, dont il convient de rappeler qu'il a été exécuté à la demande expresse de l'université flamande de Louvain ${ }^{1}$, qui l'a utilisé comme un instrument de marketing, ne garde que des souvenirs très lointains de l'ordre arborescent de jadis. Lé critère principal qui l'organise est la juxtaposition, qui est un modèle ni unitaire, ni hiérarchique. Certes, la juxtaposition n'est ici pas absolue, puisque le photographe maintient grosso modo le compartimentage des sujets selon le groupe professionnel ou sociologique auquel ils appartiennent. Pour échapper à l'arbitraire, ou pour donner un semblant d'ordre à la série d'images, l'artiste a regroupé les portraits en fonction d'un système "taxinomique" un peu fruste mais bien commode. Celui-ci, pourtant, est appliqué de manière assez lâche: tantôt la cohérence de certains groupes est assez nette, tantôt elle paraît uniquement le résultat des rapprochements personnels établis par Jacques Sonck même. De plus, et c'est là une différence absolue avec l'album de 1884, l'ordre dans lequel les sous-ensembles sont alignés devient, non pas arbitraire (encore que le fait de ne pas commencer le livre par la section des professeurs ne manque pas d'acquérir très vite une signification "politique"), mais subjectif (l'enchaînement des parties aurait pu être tout à fait autre et à l'intérieur de chacune d'elles une même liberté semble prévaloir).

Bref, le modèle juxtaposé dissout ou, si l'on préfère, dynamise le modèle arborescent. Chaque chose peut maintenant occuper plusieurs places et chaque place peut recevoir plusieurs choses. Toutefois, l'ordre choisi n'est pas non plus totalement gratuit. Le média de l'album photographique oblige en effet à opérer des choix d'ordre, qui tirent à conséquence dans la mesure où ils deviennent tout de suite des

1 Comme on l'a précisé plus haut, ce n'est pas seulement sur le plan administratif que l'université de Louvain se dédouble en 1971. L'iconographie (c'est-à-dire la politique visuelle de l'université) change également, puisque change le contexte non moins que l'imaginaire urbains des deux institutions. Du côté francophone, l'urbanisme et la sociologie très particuliers de Louvain-la-Neuve, ville (longtemps) sans habitants, se fera sentir par une insistance presque exclusive sur I'architecture. 
choix de préséance. En l'occurrence, le parcours du livre accroît l'impression de démocratisation qui se dégage déjà de la sélection plus large des sujets photographiques et du traitement plus uniforme de la manière de les photographier.

\section{La maladie chronique de l'album}

En l'espace de cent ans, l'université a donc changé d'image, et l'on aurait tort de penser que cette transformation est seulement superficielle. Même si elle est inexacte par rapport à certaines réalités moins égalitaires mais plus cachées, la représentation de soi n'est jamais qu'un ornement et son impact sur l'essence et le fonctionnement de l'institution est considérable. Pour mesurer ce genre d'effets, il importe cependant d'élargir un peu le corpus. La chose n'est point difficile : au cours du siècle qui sépare les deux albums déjà analysés, les autoreprésentations photographiques de l'université ne sont pas limitées au seul genre du portrait dont on vient de commenter deux exemples ; à bien d'autres moments de son histoire, l'université a favorisé la production d'albums très différents, dont l'étude permettra de jeter une nouvelle lumière sur les publications de 1884 et 2001 . On verra en effet que, replacées dans une trajectoire historique plus complète, les divergences entre les deux séries de portraits sont peutêtre moins grandes qu'on ne l'a suggéré jusqu'ici.

Dans le corpus des autoreprésentations photographiques de l'université au XXe siècle, il est facile d'observer la place sans cesse croissante qu'y tient la ville même de Louvain. Le traumatisme des destructions de 1914 y est sans doute pour beaucoup, puisque le feu allemand ne faisait guère de distinction entre l'alma mater et la ville, mais non moins direct est le rôle joué par l'expansion de l'université, qui oblige celle-ci à repenser de fond en comble ses rapports avec le tissu urbain qui l'entoure : gestion du patrimoine immobilier, implantation de nouveaux bâtiments "extra muros", politique d'acquisition et de construction dans un environnement qui n'est ni vierge, ni modelable à loisir (l'autre Louvain, c'est-à-dire le Louvain "civil" ou nonuniversitaire a résisté parfois aux exigences de l'institution, ne fût-ce que par sa seule présence), tous ces éléments font que l'image de l'université commence à se définir de plus en plus par rapport à son cadre humain et géographique. 
Cette nouvelle iconographie est évidemment d'abord et directement politique : après les ravages de la Première Guerre Mondiale, l'université martyre, dont les relations avec la municipalité libérale n'avaient pas toujours été simples depuis la fin du XIX $\mathrm{X}^{\mathrm{e}}$ siècle, proclame très haut ses droits moraux, voire ses droits tout court, sur les bâtiments dont elle n'est pas toujours propriétaire (c'est en effet la ville qui possède par exemple plusieurs des joyaux de l'université, dont les Halles au Drap, le siège administratif de l'université).

Mais quelles sont les autres significations de cette ouverture ? Ici encore, les apparences ne manquent pas d'induire en erreur l'observateur pressé. A première vue, on pourrait croire que la prise en considération du contexte urbain va tout à fait dans le même sens que la démocratisation relevée ci-dessus. De la même façon que l'université cesse de se réduire à son seul corps professoral, elle accepterait ainsi de communiquer avec la ville qui l'accueille et la nourrit. Dans les deux cas il y aurait, littéralement, sécularisation, voire laïcisation. Toutefois, et c'est là-dessus que l'on veut ici porter l'attention, l'intrusion de la ville soulève des défis tout à fait particuliers dans le cas précis de l'album photographique.

En effet, l'évocation d'une ville au moyen d'une série photographique suscite un problème que Jean Ricardou, évoquant la description en littérature, a qualifié de "maladie chronique"l. Le problème en question est la difficulté intrinsèque, pour tout art ou toute pratique liés au temps (comme le texte ou l'album photographiques, qui ne sont pas des accumulations ou des juxtapositions de signes, mais des suites orientées dans le temps) de disposer de manière satisfaisante une donnée fondamentalement liée à l'espace (un corps à décrire à l'aide d'un texte, une ville à déplier dans un album, notamment). Si la représentation d'une ville au moyen de la photographie n'est pas un problème en soi (il suffit de passer par une vue panoramique, par exemple), le passage du média photographique au média de l'album confronte le photographe à l'épineuse conversion d'une donnée spatiale en une donnée temporelle. Pour parer à cette difficulté, les systèmes de représentation traditionnels s'appuient généralement sur toute une gamme d'astuces ou de subterfuges. Il n'est guère utile de les détailler ici si ce n'est pour faire remarquer que les albums

1 Une maladie chronique, Paris-Bruxelles, Les Impressions Nouvelles, 1989. Les idées de l'auteur sur cette question prolongent évidemment la réflexion sur le conflit du temps et de l'espace lancée depuis au moins le XVIIIe siècle par le Laocoon de Lessing. 
photographiques de l'université mobilisent très activement ce qui constitue sans aucun doute le plus efficace de ces mécanismes de conversion spatio-temporelle : l'ajout à la couche visuelle de l'objet représenté d'une dimension chronologique que l'on sait, elle, parfaitement intégrable aux structures temporelles du média d'accueil.

Traditionnellement, cette procédure de conversion connaît deux variantes, l'une diachronique et l'autre synchronique, qui se combinent et se renforcent sans trop d'obstacles dans le cas de l'album d'images d'une ville. D'abord la mention de l'ancienneté des bâtiments et des infrastructures, qui autorise une déclinaison du corpus par tranches historiques, en l'occurrence des vestiges médiévaux aux grands chantiers de la vie future. Ensuite le tour en ville, qui contribue à diviser le bloc spatial de la ville en fonction du parcours temporel d'un visiteur plus ou moins imaginaire (par exemple de l'extérieur à l'intérieur, de l'extra muros à l'intra muros, de la vue panoramique aux vues de détail, ou inversement bien entendu, quand on passe par exemple des Halles au centre-ville au parc scientifique d'Heverlee, puis aux institutions psychiatriques de Lovenjoul, enfin à la ville nouvelle de Lovanium). Chacune de ces logiques temporelles, celle synchronique du "grand tour" (sic) comme celle diachronique du "voyage dans le temps" (resic), est solidaire de l'autre et se parcourt toujours dans les deux sens : le saut extra muros offre un regard sur l'avenir tout en permettant un retour aux racines et une nouvelle affirmation de l'identité séculaire, et ainsi de suite.

Le lien de ces imbrications spatio-temporelles avec le modèle de l'album photographique est capital. On doit constater en effet que ce modèle n'est pas le seul possible. Pendant de longues années, essentiellement dans l'entre-deux guerres, l'université lui a du reste préféré d'autres types de conjugaison d'images, celui, essentiellement, des séries de cartes postales. Or, la logique interne d'une telle série diffère radicalement de celle d'un album et ce n'est pas un hasard que les images des cartes postales sur l'université ou la ville ne ressemblent guère à celles des albums (le fait même par exemple que les deux, ville et université, y conservent une certaine autonomie, est loin d'être négligeable).

En gros, les séries de cartes postales obtempèrent à une homogénéité presque absolue : elles sont consacrées à une seule catégorie d'images, qu'elles déclinent de manière presque alphabétique (l'on sait que de tous les critères de classement l'alphabet est à la fois le plus apparemment naturel et le plus absolument arbitraire). Sans être 
arborescente comme dans l'album de 1884, leur logique unitaire n'est à aucun moment mise en question, tellement les ressemblances d'une image à l'autre effacent toute autre possibilité de structuration (il est vrai que l'ordre de ces images peut varier, mais l'essentiel est qu'aucun autre type d'organisation ne s'avère capable de menacer la série que l'on a sous les yeux : même en brouillant l'ordre des cartes postales comme si c'étaient des cartes à jouer, le résultat final serait plus ou moins analogue).

Lorsque l'université se met à publier de véritables albums photographiques, d'abord à l'occasion de l'Exposition mondiale de $1958^{1}$ (qui avait marqué le passage de la Belgique et partant, mais de manière bien plus prudente, de l'université aux temps modernes de l'âge atomique), puis au moment des années post- $68^{2}$ (qui avaient signifié la scission administrative et géographique de l'université bilingue en deux universités unilingues, séparées désormais par la langue comme par les kilomètres), l'abandon du modèle de la carte postale au profit de celui du livre ne se réduit pas seulement à une question de formule, le livre étant jugé par exemple plus "moderne" que la carte postale ou mieux approprié à la reproduction d'images plus spectaculaires et d'un dessein plus esthétique. Contrairement aux séries de cartes postales, la forme et le contenu semblent en effet presque chaotiques, ou au moins d'une diversité jusque-là inconnue, un peu comme si le bruit et la fureur du "siècle" éclataient à la figure de l'alma mater.

\section{L’album comme bouée de sauvetage}

La raison fondamentale du passage de la carte postale à l'album ne tient pas seulement à cet aggiornamento stylistique. Elle est au contraire idéologique et en rapport direct avec le phénomène de sécularisation décrit plus haut et surtout avec les efforts des autorités universitaires de maîtriser et de canaliser les effets perturbateurs de ce phénomène. L'album permet en effet d'ouvrir la représentation de l'université à la vie extérieure qui fait pression de toutes parts en même temps qu'il aide à canaliser les mutations à l'aide des nouveaux

1 Valentin DENIS, Katholieke Universiteit te Leuven 1425-1958, Leuven, 1958, publié par l'université.

2 Traditie en toekomst van een universiteit : Leuven. (Foto's van W. De Mulder, W. François en R. Van den Boom), Lannoo, Tielt/Utrecht, 1970. 
principes structurants apportés par la ville de Louvain (au sens très large du terme, qui comprend toutes les facettes de l'inscription de l'université dans un contexte matériel donné).

Pour qui feuillette même rapidement les albums de la période 1958-1970, il est patent que dans ces années-là l'ordre traditionnel du discours photographique s'effondre. S'il reste encore quelques traces de la "grande arborescence" de 1884 dans l'album de 1958, force est de constater que le modèle émergent est celui de la mosaïque, qui se rapproche en 1970 du modèle du puzzle ${ }^{1}$. De manière moins métaphorique, on pourrait dire que ce qui tend à l'évanescence dans ces albums, ce sont d'une part la division nette entre les différentes parties de l'ensemble (les albums favorisent les transitions fluides et le décloisonnement généralisé) et d'autre part la cohérence interne des parties prises isolément (les albums multiplient franchement les formes d'hétérogénéité à l'intérieur de chaque classe ou de chaque catégorie, ce qui se voit d'autant mieux que l'on reste malgré tout encore loin du modèle de la juxtaposition presque intégrale à la Sonck). Pour l'album de 1970, la transgression des limites est certes plus marquée que dans celui de 1958, mais elle n'est pas entièrement neuve: relus à la lumière de ce qui s'impose massivement en 1970, les changements de 1958 apparaissent déjà comme "prophétiques"2.

En même temps, toutefois, ni le livre de 1958, ni le complément de 1970, ne s'avèrent désorganisés, loin de là. L'intrusion des corps étrangers que sont les éléments non traditionnellement universitaires (la ville d'un côté, les étudiants de l'autre, l'ancienne université se réduisant à ses professeurs et à ses propres bâtiments) est au contraire mise à contribution pour rétablir un ordre nouveau, certes plus complexe que l'ordre ancien, mais qui n'en est pas moins un ordre rassurant. L'expansion spatiale du cadre de l'université et l'affirmation de la vie estudiantine sont mobilisées pour pallier les insuffisances de l'ordre ancien. A la structure arborescente traditionnelle se substitue une structure plus fluide, dont les principes d'organisation

1 Pour l'importance heuristique et théorique de ces deux métaphores, voir le livre de Lucien DÄllenbaCh, Mosaiques, Paris, Éd. du Seuil, 2001.

2 Ici encore, une analyse plus fouillée serait nécessaire pour bien marquer les signes d'un changement qui est a été moins abrupte qu'on ne le pense aujourd'hui. Une place importante devrait être donnée à cet égard au petit "supplément" de la publication de 1958, qui sort en 1966 et où l'on remarque clairement l'intrusion de plusieurs signes de la modernité, dont par exemple la présence très manifeste des deux "prorecteurs", manifestation de fait d'une scission linguistique en voie d'accomplissement. 
ne laissent pas de dégager rapidement une logique nouvelle, elle fortement temporalisée. A l'image de la société civile, l'université se pense désormais dans le temps : solidement ancrée dans le passé comme la ville médiévale de Louvain, orgueilleusement tournée vers l'avenir à l'image de ses étudiants venus du monde entier.

La forme de l'album photographique, qui autorise la séquentialisation du modèle des cartes postales, convient à merveille à cette logique de re-signification par temporalités complexes interposées. Faisant coïncider feuillètement des pages et voyage dans le temps, l'album arrive à étouffer les tensions entre l'universitaire et le nonuniversitaire, entre l'institutionnel et le non-institutionnel, entre la tradition et la modernité, entre l'identité et l'altérité.

Dans cette perspective, le retour final, dans le travail de Jacques Sonck en 2001, à une forme d'album qui refoule autant que possible la dimension temporelle de ses images comme la plus-value chronologique du feuillètement, est une évolution très intrigante. Ce qui articule ce nouveau livre en sous-main est la logique de la série des cartes postales, c'est-à-dire la logique qui détemporalise le plus possible et le sujet et la forme de sa présentation au cour d'un livre. Si le livre est encore trop près de nous pour qu'il soit possible d'en donner une interprétation correcte, il n'est pas absurde de supposer déjà que ce retrait de l'histoire témoigne d'un rapport inédit entre l'université et son contexte. Que l'album ne soit plus utilisé comme un instrument de neutralisation des tensions entre l'institution et la société (le "siècle", comme le dirait le langage ancestral de Louvain), en dit sans doute très long sur la pacification qui a eu lieu et sur l'intégration de l'université à un contexte social, géographique, politique et autre, dont l'existence même a longtemps été soit ignorée, soit ressentie comme une menace. 